Annuaire suisse de politique de développement

$19 \mid 2000$

Pour une politique plus cohérente envers les pays en développement

\title{
La place des droits humains dans les relations Nord-Sud de la Confédération : le problème de la cohérence
}

Jean-Claude Huot

\section{CpenEdition}

Journals

Édition électronique

URL : https://journals.openedition.org/aspd/834

DOI : 10.4000/aspd.834

ISSN : 1663-9669

Éditeur

Institut de hautes études internationales et du développement

Édition imprimée

Date de publication : 1 avril 2000

Pagination : $37-51$

ISSN : 1660-5934

Référence électronique

Jean-Claude Huot, «La place des droits humains dans les relations Nord-Sud de la Confédération : le problème de la cohérence », Annuaire suisse de politique de développement [En ligne], 19 | 2000, mis en ligne le 15 août 2012, consulté le 21 septembre 2021. URL : http://journals.openedition.org/aspd/834 ; DOI : https://doi.org/10.4000/aspd.834 


\title{
LA PLACE DES DROITS HUMAINS DANS LES RELATIONS NORD- SUD DE LA CONFÉDÉRATION: LE PROBLĖME DE LA COHÉRENCE
}

\author{
Jean-Claude Huot*
}

L

E 22 SEPTEMBRE 1986, le Conseil fédéral publiait une déclaration dans laquelle il refusait d'adopter des sanctions économiques envers l'Afrique du Sud. Il expliquait qu'il avait plafonné dès 1974 les exportations de capitaux et qu'il allait opérer une "surveillance statistique» des relations économiques avec l'Afrique du Sud afin d'éviter que la Suisse serve de plaque tournante pour esquiver les sanctions décidées sur le plan international. En 1999, une étude non gouvernementale ${ }^{1}$ démontre que ces mesures ont été facilement contournées, et une enquête journalistique met le doigt sur les relations entre les services secrets suisses et sud-africains. Pourtant, à la même époque, la Suisse soutenait des organisations sud-africaines engagées en faveur des droits de l'homme et du droit de vote de la population noire sud-africaine. Elle a également soutenu entre 1987 et 1989 l'initiative secrète d'un avocat sud-africain visant à faire libérer Nelson Mandela et à favoriser le dialogue entre l'ANC et le gouvernement sud-africain ${ }^{2}$.

Dans une interview accordée à L'Hebdo le 8 avril 1999, l'ancien conseiller fédéral René Felber expliquait: «Des collaborateurs m'avaient informé que, vraisemblablement, les limites de crédit que nous avions fixées étaient dépassées. Au Conseil fédéral, quand je disais cela, on me répondait que le service économique chargé de la surveillance faisait des contrôles mais qu'ils étaient difficiles. On m'assurait aussi qu'on mettait les banques en garde. En fait, je crois que nous avons fait preuve de laxisme.»

Ainsi se présentait la politique suisse envers le régime de l'apartheid en Afrique du Sud. Chaque département fédéral menait sa propre politique sans qu'il y ait de stratégie d'ensemble. Par absence d'une vision globale et d'instruments permettant de donner à l'action gouvernementale une direction collective, la politique de la Confédération face au régime de l'apartheid était incohérente.

C'était dans les années 80. Aujourd'hui, qu'en est-il de cette cohérence entre les droits humains et les relations que la Suisse entretient avec le reste du monde, et plus particulièrement avec les pays du Sud? Pour répondre à cette question je vais commencer par situer la problématique de la cohérence par rapport à la complexité du monde actuel. Puis j'examinerai l'évolution de la politique fédérale concernant la cohérence dans le domaine des droits de l'homme ainsi que deux champs de tension qui apparaissent. Je terminerai par quelques propositions permettant d'accélérer la recherche de cohérence en cours.

Secrétaire de la commission nationale suisse Justice et Paix, organe consultatif de la Conférence des évêques suisses sur les questions politiques, sociales, économiques et éthiques, Berne.

1. M. Madörin, G. Wellmer und M. Egli, Apartheidschulden, der Anteil Deutschlands und der Schweiz, Basel, Februar 1999.

2. Voir Les relations entre la Suisse et l'Afrique du Sud, rapport du groupe de travail interdépartemental Suisse-Afrique du Sud, Berne, juillet 1999. 


\section{DE QUELLE COHÉRENCE PARLONS-NOUS?}

\section{$\square$ Le principe de "rétinité»}

S'il est un domaine complexe, c'est bien celui de la politique étrangère. La multiplicité des acteurs (Etats, organisations internationales gouvernementales et non gouvernementales, entreprises), la diversité de leurs objectifs, les divers domaines d'intervention font qu'il est difficile de mener une action cohérente. L'exemple de l'attitude de la Suisse face au régime de l'apartheid le montre bien. Il y a eu incohérence car le Conseil fédéral ne s'est pas donné les moyens d'avoir une vue d'ensemble. Pour pallier un tel aveuglement, il est nécessaire de comprendre les implications des décisions prises dans toute leur complexité. C'est ce que permet d'élaborer un principe de connaissance que l'on peut nommer de « rétinité » ${ }^{3}$ (du latin rete, «filet»).

Ce principe est né de la discussion sur l'éthique environnementale. La perception croissante de l'impact des activités humaines sur l'environnement a rendu nécessaire la réintégration du monde culturel, y compris de la dynamique déterminante de l'économie, dans les régulations de l'écosystème ${ }^{4}$, au niveau tant de la pensée que de l'action. Ainsi le principe de «rétinité » exige de relier dans un même élan les dimensions économiques, sociales et écologiques du développement. Selon ce principe, les mesures prises dans le domaine du développement économique ne peuvent pas être conçues sans qu'il soit mené en parallèle un examen des composantes sociales et écologiques des projets soutenus. Réciproquement, des projets qui visent le développement social, que ce soit dans les domaines de la santé, de l'éducation ou de la promotion de la femme, doivent prendre en compte les impacts prévisibles sur le développement économique et sur l'environnement.

Comme principe, la «rétinité » va au-delà du domaine des relations hommenature, car elle suppose une interrelation étroite entre les divers champs d'activités humaines et entre ceux-ci et l'environnement. Son usage permet d'éviter d'avoir des relations commerciales avec un pays sans prendre en considération les autres dimensions des relations avec ce pays, sans tenir compte de l'impact social et environnemental sur le respect des droits humains. Il est tout aussi impossible de mener un dialogue politique avec un Etat sans tenir compte des impacts que ce dialogue peut avoir sur les autres aspects des relations entretenues avec lui'.

\section{$\square$ Un discernement nécessaire}

Le monde est devenu multipolaire, le poids des relations économiques et financières à l'échelle planétaire établit de fait des interrelations étroites entre les sociétés du monde entier. Si cette mondialisation en cours est inéluctable, elle ne se limite pas pour autant aux phénomènes économiques. Les cultures des

3. Der Rat von Sachverständigen für Umweltfragen, Umweltgutachten 1994, Stuttgart, 1994, cité par Hans J. Münk, «Welche Zukunft wollen wir», Schweizerische Kirchenzeitung, 8/1999 p. 114

4. Die deutschen Bischöfe - Kommission für gesellschaftliche und soziale Fragen, Handeln für die Zukunft der Schöpfung, Bonn, 1998, p. 60.

5. Les auteurs utilisant ce concept de «rétinité » qualifient le principe ainsi développé d'éthique. Je ne le fais pas dans le présent article, estimant que ce principe n'établit pas de hiérarchie entre les interrelations envisagées mais postule uniquement la nécessité de tenir compte de ces interrelations. On se trouve à mon sens davantage au niveau méthodologique qu'éthique. 
diverses nations sont en train de changer. Les préoccupations écologiques font redécouvrir aux cultures occidentales le lien étroit qui unit la culture à la nature en même temps qu'elles permettent de réintégrer les cultures d'autres régions du monde dans le patrimoine commun de l'humanité; l'alliance entre les télécommunications et l'ordinateur, concrétisée par le réseau Internet et le courrier électronique, permet une communication humaine qui dépasse les frontières géographiques et culturelles. Ainsi naît une «nation» mondiale dans laquelle les habitants de la terre entière prennent progressivement conscience du destin commun qui les unit entre eux et avec la biosphère qu'est la Terre.

Cette «nation» mondiale dispose de sa charte, d'instruments pour la mettre en œuvre et de programmes d'action. La charte est bien sûr celle des Nations Unies qui, avec la Déclaration universelle des droits de l'homme, indique l'aspiration à la paix, au respect des droits humains découlant de la dignité inhérente à chaque personne humaine. Les instruments sont constitués par l'ensemble des pactes et conventions relatifs non seulement aux droits de l'homme, mais également à l'environnement. Enfin, les programmes sont inscrits dans les résultats des diverses conférences mondiales organisées depuis le début des années 90.

\section{Conférences mondiales organisées au cours des années 90}

1990 Conférence mondiale sur les droits de l'enfant, New York
1992 Conférence des Nations Unies sur l'environnement et le développement
("Sommet de la Terre »), Rio de Janeiro
1993 Conférence mondiale sur les droits de l'homme, Vienne
1994 Conférence internationale sur la population et le développement, Le Caire
1995 Conférence mondiale sur les femmes, Pékin
1995 Sommet mondial pour le développement social ("Sommet social »), Copenhague
1996 Conférence mondiale sur les établissements humains ("Habitat II"), Istanbul
1996 Sommet mondial pour l'alimentation, Rome (FAO)

Source: Rapport du Conseil fédéral sur les relations entre la Suisse et l'Organisation des Nations Unies du 1" juillet 1998, chiffre 222

Les résultats de ces conférences peuvent paraître limités tant la distance reste grande entre les déclarations d'intention et la pratique. Toutefois, elles permettent aux Etats et aux acteurs de la société civile d'aborder les problèmes communs à toute l'humanité d'une manière globale et expriment une volonté de trouver des bases communes d'action au-delà des intérêts spécifiques à chaque pays. C'est ainsi que, malgré la réticence de certains gouvernements, la Déclaration de la Conférence mondiale de Vienne (1993) souligne que «tous les droits de l'homme sont universels, indissociables, interdépendants et intimement liés», que «la démocratie, le développement et le respect des droits de l'homme et des libertés fondamentales sont interdépendants et se renforcent mutuellement ${ }^{6}$.

6. Déclaration et programme d'action de Vienne, Conférence mondiale sur les droits de l'homme, Vienne, 14-25 juin 1993 , chiffres 5 et 8 . 


\section{$\square$ Vers un Etat de droit à l'échelle planétaire}

Avec l'arrestation d'Augusto Pinochet en mars 1999, l'inculpation de Slobodan Milosevic par le Tribunal pénal international pour l'ex-Yougoslavie en mai de la même année et l'approbation des statuts de la Cour pénale internationale en juillet 1998 à Rome est apparu sur la scène internationale un nouvel acteur: une justice aspirant à l'indépendance. La séparation des pouvoirs est une des caractéristiques essentielle de l'Etat de droit. Or, sur le plan mondial, on en est encore loin. Tout reste encore du ressort des gouvernements, qui négocient entre eux les obligations auxquelles ils acceptent de se soumettre avec plus ou moins de bonne volonté. Les tribunaux créés pour l'ex-Yougoslavie et le Rwanda dépendent encore des décisions prises par le Conseil de sécurité des Nations Unies, donc d'une décision des gouvernements. Avec la Cour pénale internationale ce sera moins le cas puisque les compétences de cette Cour sont définies dans ses statuts. Il est d'ailleurs significatif que ce soit justement l'étendue de ces compétences qui pose encore le plus de problèmes.

Kofi Annan relevait à l'ouverture de l'Assemblée annuelle des Nations Unies en septembre 1999: «La notion de stricte et traditionnelle souveraineté ne répond plus aux aspirations des peuples à jouir des libertés fondamentales. » ${ }^{7} \mathrm{Il}$ apparaît de plus en plus clairement que, de la même manière qu'une justice indépendante au niveau des Etats garantit aux individus le respect de leurs libertés, une justice indépendante sur le plan international est le garant de ces mêmes libertés audelà des frontières nationales.

De même, il est nécessaire qu'une société civile se mondialise, comme l'a fait l'économie. Le poids croissant des organisations non gouvernementales sur la scène internationale est à cet égard réjouissant. En témoigne le processus qui a conduit à l'approbation en décembre 1997 de l'accord d'Ottawa interdisant les mines antipersonnel. L'action d'organisations non gouvernementales de la planète entière a poussé une majorité de gouvernements à accepter une telle interdiction. Pareillement, les millions de signatures remises en juin 1999 à Cologne ne sont certainement pas étrangères au fait que le FMI vend une partie de ses réserves d'or permettant d'élargir à 36 pays les opérations de désendettement envisagées. Ainsi, le champ d'action des citoyens et citoyennes déborde de plus en plus les frontières du pays dont ils ont le passeport, et commence à prendre les dimensions de la planète entière, contribuant ainsi à l'émergence d'une «citoyenneté mondiale».

Dans un tel contexte, la cohérence de la politique étrangère de la Confédération n'est pas seulement une nécessité logique, demandant à la main droite de connaître ce que fait la main gauche et à celui qui agit aujourd'hui de penser à ce qu'il a fait la veille; elle apparaît comme la nécessité de s'approprier les dynamiques du monde environnant afin d'y faire valoir ses propres objectifs de manière efficace et crédible. Partager les valeurs fondamentales communes à l'humanité, participer à l'émergence d'un Etat de droit sur le plan mondial et user du principe de «rétinité» deviennent des exigences si la Suisse veut être entendue. 


\title{
2. LA SUISSE EN RECHERCHE DE COHÉRENCE
}

\section{$\square$ L'ancrage des droits humains dans la politique fédérale}

Depuis la publication de son rapport sur la politique suisse en faveur des droits de l'homme de 1982, le Conseil fédéral a donné de fortes impulsions pour enfin intégrer la Suisse au système international de protection des droits humains. La Confédération a ratifié les principaux instruments de défense des droits humains, notamment les pactes I (droits économiques, sociaux et culturels) et II (droits civils et politiques) entrés en vigueur le 18 septembre 1992. Encore en 1999, le Conseil fédéral a adressé aux Chambres son message relatif à la Convention pour la prévention et la répression du crime de génocide du 9 décembre 1948 et à la révision correspondante du droit pénal, ouvrant ainsi la procédure de ratification. Avec cette dernière ratification, on peut dire que la Suisse aura adhéré aux principaux instruments internationaux. Il manque encore la Convention internationale sur la protection des droits de tous les travailleurs migrants et des membres de leur famille de 1990 ainsi que les statuts de la Cour pénale internationale de 1998.

\section{Chronologies des dates de ratification ou d'adhésion de la Suisse aux pactes ou conventions internationaux}

\author{
2.12.1986 Convention du 10 décembre 1984 contre la torture \\ et autres peines ou traitements cruels, inhumains ou dégradants \\ 18.6.1992 Pacte international du 16 décembre 1966 \\ relatif aux droits économiques, sociaux et culturels \\ 18.6.1992 Pacte international du 16 décembre 1966 relatif aux droits civils et politiques \\ 16.6.1994 Deuxième Protocole facultatif se rapportant au Pacte international relatif aux droits \\ civils et politiques, visant à abolir la peine de mort, du 15 décembre 1989 \\ 29.11.1994 Convention internationale du 21 décembre 1965 \\ sur l'élimination de toutes les formes de discrimination raciale \\ 24.2.1997 \\ Convention du 20 décembre 1989 relative aux droits de l'enfant \\ 27.3.1997 \\ Convention du 18 décembre 1979 sur l'élimination \\ de toutes les formes de discrimination à l'égard des femmes \\ Procédure de Convention du 9 décembre 1948 pour la prévention \\ ratification en cours et la répression du crime de génocide
}

Source: Rapport du Conseil fédéral sur les relations entre la Suisse et I'Organisation des Nations Unies du 1er juillet 1998, annexe 4.

Mais les ratifications ne suffisent pas. Il est remarquable que ce rattrapage se soit inscrit dans le cadre d'une formulation précise des objectifs de la politique étrangère. Ces objectifs, contenus dans le Rapport du Conseil fédéral sur la politique extérieure de la Suisse dans les années 90 du 29 novembre 1993, sont les suivants:

- le maintien et la promotion de la sécurité et de la paix;

- l'engagement en faveur des droits de l'homme, de la démocratie et des principes de l'Etat de droit; 
- l'accroissement de la prospérité commune;

- la promotion de la cohésion sociale;

- la préservation du milieu naturel.

L'impact de ce rapport a été double. D'une part, le Conseil fédéral a approuvé dans le même mouvement les Lignes directrices Nord-Sud ${ }^{8}$, rapport dans lequel le gouvernement développe les conséquences des objectifs généraux pour la coopération au développement. Suite à ces Lignes directrices, la Direction du développement et de la coopération (DDC) a publié une série de documents traçant les pistes concrètes de son action pour la réalisation de ces objectifs?. D'autre part, la révision totale de la Constitution fédérale a permis d'inscrire dans la charte fondamentale de notre pays les objectifs précédemment approuvés par le Conseil fédéral: «La Confédération s'attache à préserver l'indépendance et la prospérité de la Suisse; elle contribue notamment à soulager les populations dans le besoin et à lutter contre la pauvreté ainsi qu'à promouvoir le respect des droits de l'homme, la démocratie, la coexistence pacifique des peuples et la préservation des ressources naturelles. $\gg^{10}$

Ainsi, tant sur le plan interne que face aux obligations internationales, la Confédération dispose des instruments nécessaires pour prendre plus largement en compte les «critères des droits de l'homme, de la démocratie et de l'Etat de droit pour des décisions relevant de la politique générale (politique d'asile, politique économique extérieure, politique de développement, etc.). ${ }^{11}$

\section{$\square$ Les droits de l'homme dans les relations Nord-Sud}

Selon les Lignes directrices Nord-Sud, la promotion du respect des droits humains, de la démocratie et de l'Etat de droit passe par trois degrés d'interventions: les mesures positives (soutien à des organisations et institutions à même d'améliorer la situation dans le pays partenaire), le dialogue politique ainsi que la conditionnalité directe ${ }^{12}$.

Les Lignes directrices que s'est données la DDC mettent l'accent sur les mesures positives. La DDC part de l'idée que «la réalisation des droits de l'homme doit être un but du processus de développement, que le respect des droits de l'homme fait partie des conditions favorables à un tel processus, qu'un respect minimal des libertés et droits fondamentaux est une condition préalable indispensable à un processus de développement et enfin que le développement peut contribuer de façon considérable à la réalisation des droits de l'homme $»^{13}$. La double redondance de cette phrase souligne l'interdépendance entre le développement et le respect des droits humains que perçoivent les responsables de la coopération au développement. A leurs yeux, leur travail participe à la promo-

8. Rapport du Conseil fédéral sur les relations Nord-Sud de la Suisse dans les années $90 \mathrm{du} 7$ mars 1994, dit «Lignes directrices Nord-Sud».

9. Promotion des droits de l'homme et coopération au développement. Lignes directrices adoptées par la DDC le 24 février 1997; Le concept d'Etat de droit et ses implications pour la coopération au développement, octobre 1998; et d'autre part les Ecrits sur le développement: $\mathrm{n}^{\circ} 1$, Le développement social dans les relations Nord-Sud, avril 1997, n², Décentralisation et développement, janvier 1999.

10. Art. 54 de la nouvelle Constitution fédérale.

11. Rapport sur la politique extérieure de la Suisse dans les années 90 du 29 novembre 1993, chiffre 4.1.2.

12. Lignes directrices Nord-Sud, chiffre 211.

13. Promotion des droits de l'homme et coopération au développement. Lignes directrices adoptées par la DDC le 24 février 1997 (ci-après Promotion des droits de l'homme), chiffre I.2. 
tion des droits économiques, sociaux et culturels. Mais ils soulignent, en relevant qu'il s'agit là d'une préoccupation plus récente, que les droits civils et politiques et le respect des libertés fondamentales «permettent aux individus de disposer de meilleurs instruments pour améliorer leur condition et ouvrent l'espace indispensable pour influencer l'action des pouvoirs publics $»^{14}$. Des notions telles que ownership - exprimant l'idée que le processus de développement est vécu et activé par les personnes concernées qui ainsi s'approprient ledit processus - et empowerment - selon lequel la coopération doit aider les individus et les groupes bénéficiaires à acquérir la maîtrise de leur propre développement - sont entrées dans le vocabulaire de la coopération. Elles ont contribué à renforcer l'idée selon laquelle les droits humains et le développement sont deux dimensions d'un même processus. La coopération au développement reçoit de cette façon une nouvelle légitimation, celle des droits humains. Il n'est donc pas étonnant qu'en déterminant les dix principes stratégiques de son action dans ce domaine, la DDC place les mesures positives en tête de liste. Elle est en charge de ce type de mesures.

Le dialogue politique échappe quant à lui plus largement à la coopération au développement, même si les liens tissés dans le cadre de celle-ci facilitent grandement le dialogue, donnant à la Confédération une plus grande crédibilité auprès de ses interlocuteurs.

La conditionnalité doit, selon la DDC, être mise en œuvre de manière différenciée en fonction de la situation spécifique du pays partenaire. En cas de doute sur la volonté du gouvernement d'améliorer la situation, il est envisagé de procéder par étapes et de faire dépendre la poursuite du programme d'aide d'une évaluation régulière (tous les trois ou six mois) des progrès réalisés. En cas de violation systématique des droits de l'homme ou de conditions-cadres insuffisantes, la DDC cherche à établir des relations avec des secteurs non gouvernementaux afin de renforcer les capacités de la société civile. Elle estime alors que le gouvernement ne peut plus être considéré comme un partenaire de la coopération à long terme. Ce fut par exemple le cas avec Madagascar en 1998. Le pays a été sorti de la liste des pays de concentration à cause d'un manque de clarté dans l'enquête menée sur le meurtre d'un responsable de projets et parce que les efforts du gouvernement pour combattre la pauvreté n'ont pas paru assez convaincants à la DDC. Actuellement, les projets sont revus et il est prévu d'apporter un soutien à la société civile à travers des œuvres d'entraide en Suisse.

En dernière extrémité, une interruption de la coopération est envisagée. Mais il s'agit alors d'une décision politique pour laquelle le Conseil fédéral s'est récemment déclaré seul compétent «afin, explique-t-il, d'assurer la cohérence de la politique extérieure de la Suisse [...] et pour préserver la crédibilité des objectifs de politique extérieure». Les raisons justifiant une telle interruption sont «des efforts insuffisants quant à l'application du principe de "good governance", des violations graves des droits de l'homme, la suspension de processus de démocratisation, des atteintes importantes à la paix et à la sécurité, l'absence de volonté d'accepter le retour sur son territoire de ses propres ressortissants » ${ }^{15}$.

14. Promotion des droits de l'homme, chiffre II.5.

15. Communiqué du Conseil fédéral du 20 septembre 1999. Voir aussi à ce sujet Message concernant la continuation de la coopération technique et l'aide financière en faveur des pays en développement du 7 décembre 1998, chiffre 431.5; Dix ans de coopération internationale de la Suisse 1986-1995, chiffre 223; Promotion des droits de l'homme, chiffres III.4 et IV.3. 


\section{$\square$ Le leitmotiv de la cohérence}

«Le cloisonnement traditionnel entre les divers domaines politiques, tels que l'écologie, l'économie, le commerce et le développement, ne permet plus de résoudre, avec l'efficacité requise, les problèmes qui se posent. ${ }^{16}$ Dans son message de 1998, le Conseil fédéral reprend la demande des Lignes directrices Nord-Sud: il faut une «politique cohérente envers le Sud». «Formuler une telle politique revient à montrer les contradictions éventuelles entre des intérêts nationaux à court terme et les buts de la politique suisse de développement, puis à les intégrer, de façon aussi transparente que possible, dans les processus de décisions politiques. ${ }^{17}$ Le rapport sur les années 1986-95 de la coopération internationale de la Suisse va encore plus loin: "Leur mise en application [des Lignes directrices Nord-Sud] requerra des décisions politiques qui, dans notre propre intérêt, accorderont plus de poids à la garantie de l'existence à long terme qu'à nos intérêts nationaux à courte échéance. $»^{18}$

Sur cette base, la DDC peut définir sa stratégie dans ses propres Lignes directrices relatives à la promotion des droits humains. Elle reconnaît toutefois que l'engagement en faveur des droits de l'homme constitue une affaire politiquement délicate, impliquant une prise de risque qui doit être calculée ${ }^{19}$. Elle souligne son effort pour procéder par programme dans le pays partenaire plutôt que par projet, ainsi que la nécessité d'une coordination internationale. Elle s'oblige également à examiner «chaque projet et chaque programme pour identifier leurs éventuelles incidences négatives sur la situation des droits de l'homme», en ajoutant: «Il convient de vouer une attention particulière aux grands projets d'infrastructure (déplacements de population, etc.). $\gg^{20}$ Enfin, le dernier principe stratégique qu'elle se donne est celui de la cohérence, dans lequel elle indique que le besoin de cohérence englobe les actions du DFAE dans son ensemble, les mesures engagées par la coopération suisse, y compris celles qui relèvent de la politique économique et commerciale, la promotion des exportations et des investissements et d'autres domaines comme l'aide au retour des réfugiés et des requérants d'asile, la politique relative aux migrations ainsi que les exportations de matériel de guerre.

Ces textes semblent dégager une certaine clarté. D'un côté, la nécessité de dépasser les intérêts nationaux à court terme dans l'idée que la prospérité de la population suisse «dépend à long terme du destin du Sud $»^{21}$; de l'autre, l'indication précise des divers domaines de l'action gouvernementale qui doivent être unis par des objectifs cohérents «si l'on veut que celle-ci s'inscrive dans une politique des droits de l'homme crédible et efficace $»^{22}$.

Toutefois, cette clarté ne se retrouve pas dans la plate-forme de la politique extérieure de notre pays. Quand le Rapport sur la politique extérieure des années 90 aborde les questions de cohérence (chiffre 4.3), le critère des droits de l'homme n'apparaît plus. La cohérence est exigée par «la nécessité de pré-

16. Message concernant la continuation de la coopération technique et l'aide financière en faveur des pays en développement du 7 décembre 1998, chiffre 211.

17. Lignes directrices Nord-Sud, chiffre 14

18. Dix ans de coopération internationale de la Suisse 1986-1995, chiffre 46.

19. Promotion des droits de l'homme, principe stratégique 5.

20. Promotion des droits de l'homme, chiffre III.9 (principe stratégique 9).

21. Lignes directrices Nord-Sud, chiffre 14.

22. Promotion des droits de l'homme, chiffre III.10. 
server l'attrait de la place financière et économique que constitue la Suisse, le souci d'assurer une utilisation aussi efficace que possible de nos moyens limités, la volonté de faire valoir au mieux nos intérêts». Le Conseil fédéral souhaite une meilleure coordination entre la politique extérieure et la politique intérieure, entre les divers domaines de la politique extérieure et entre les objectifs et instruments de cette dernière. En dernière instance, il rappelle qu'il lui appartient d'assumer «la responsabilité de la cohérence de la politique extérieure de la Suisse». Mais il ne tranche pas; il constate que «la formulation d'une politique extérieure cohérente est rendue malaisée par les fréquentes contradictions entre ses divers objectifs, de même qu'entre les moyens de les atteindre» et il explique que «objectifs et instruments présentent tous, par principe, une importance égale».

$\mathrm{Au}$ vu de cette hésitation, il n'est pas étonnant que le Conseil fédéral précise ensuite dans les Lignes directrices Nord-Sud où il voit des risques d'incohérence. Celles-ci mettent le doigt sur les conflits d'intérêts qui peuvent surgir entre la promotion de l'emploi et des exportations d'une part, la politique en faveur des droits humains d'autre part. Il en découle l'exigence suivante: «Dans l'appréciation des risques en vue de l'octroi de la garantie aux risques à l'exportation (GRE) pour des livraisons dans les pays en développement les plus pauvres, il est nécessaire de mettre plus l'accent sur les aspects d'ordre politique et sur le respect des droits de l'homme dans les pays récipiendaires. ${ }^{23}$ Dans un autre domaine, celui des migrations, elles font de même: «Il faut cependant rester attentif au conflit d'intérêts potentiel entre, d'une part, les mesures préventives et les moyens mis en œuvre dans les pays d'origine des migrants du point de vue de la politique de migration et, d'autre part, les objectifs de la coopération au développement. $»^{24}$ S'ensuit un appel à la cohérence entre les politiques d'immigration, d'asile et de coopération au développement afin que les conditions économiques et politiques permettent aux populations concernées de vivre décemment dans leur région d'origine ${ }^{25}$. Ces deux domaines méritent donc un examen plus attentif.

\section{DEUX CHAMPS DE TENSION}

\section{$\square$ La politique économique extérieure}

Le cas très connu du barrage des Trois-Gorges en Chine illustre bien la difficulté de mener une politique cohérente dès qu'il s'agit de relations économiques. ABB a trois contrats de livraison pour ce gigantesque barrage. Pour deux d'entre eux, elle bénéfice de la garantie contre les risques à l'exportation (GRE). La première garantie date de 1997; la seconde a reçu l'aval de la commission de la GRE en septembre $1999^{26}$. La Déclaration de Berne, qui suit de près les activités de la GRE, a protesté contre cette décision, constatant que ce barrage va occasionner le déplacement de 2 millions de personnes et provoquer de massives destructions environnementales ${ }^{27}$.

23. Lignes directrices Nord-Sud, chiffre 213.

24. Lignes directrices Nord-Sud, chiffre 222.

25. Lignes directrices Nord-Sud, chiffre 231.

26. NZZ, 21.9.99.

27. Neue Exportrisikogarantie für das Drei-Schuchten-Projekt, communiqué de presse de la Déclaration de Berne du 20 septembre 1999. 
Est-ce conforme aux principes fondamentaux de la politique suisse en matière d'aide au développement, comme l'exige la loi ?28 Est-ce compatible avec les cinq objectifs de la politique extérieure de la Confédération? On peut en douter tant les conséquences de la construction de ce barrage sont lourdes. Cela a d'ailleurs provoqué un débat intense, en Chine comme dans le reste du monde. La Banque mondiale s'est elle-même retirée du projet. Mais l'objectif de la GRE est de soutenir les exportations en offrant aux entreprises une assurance contre certains risques. Qu'il soit ainsi possible d'ignorer les objectifs du gouvernement d'une part, d'interpréter la loi d'une manière pour le moins extensive d'autre part, illustre un fait très simple: l'économie est un domaine autonome et la politique économique extérieure ne doit pas être soumise aux critères de la politique extérieure générale. Ainsi, le constat posé en 1992 reste actuel: la Suisse «ne prend pas le respect des droits de l'homme comme critère de ses relations économiques avec 1'étranger ${ }^{29}$. Il suffit d'ouvrir un rapport de la GRE pour le constater: l'impact que pourraient avoir les exportations soutenues sur les pays partenaires n'est pas évoqué une seule fois; la rationalité ainsi développée est instrumentale, elle ne s'interroge par sur les fins, mais vise l'efficacité: aider à exporter.

Cette manière de comprendre l'économie est courante. Celle-ci a ses lois propres, impératives. L'interférence de valeurs sociales, de règles propres à d'autres domaines de la vie humaine, est considérée comme perturbatrice pour le bon fonctionnement de l'économie. Il ne faut pas mélanger la politique étrangère en charge des questions des droits humains avec la politique économique afin d'éviter d'imposer à la politique économique des règles qui lui sont étrangères ${ }^{30}$.

L'économie, comme le relève d'ailleurs Franz Blankart, n'est toutefois pas neutre du point de vue des droits de l'homme. Elle reste, qu'on le veuille ou non, une des dimensions de l'espace public et n'est pas un jeu séparé ${ }^{31}$. Il doit donc être possible de trouver un terrain de croisement entre la logique économique et la logique des droits de l'homme. Mais cela suppose que les règles découlant des droits humains soient intégrées dans l'action économique au même titre qu'entre maintenant dans la pratique économique le respect de l'environnement. En conséquence, les interventions de l'Etat dans le domaine économique devraient être examinées quant à leur impact sur les droits humains de la même manière qu'elles commencent à l'être concernant l'environnement. Ainsi, la triade des dimensions économique, sociale et environnementale définissant le principe de «rétinité » peut être prise en compte. Et la conformité de la pratique à la définition du développement durable donnée par le $\mathrm{Seco}^{32}$ luimême devient possible.

28. Loi fédérale sur la garantie contre les risques à l'exportation, art. 1, al. 2, en vigueur depuis le $1^{\text {er }}$ février 1981.

29. M.-C. Kraft et J.-D. Vigny, «La politique suisse à l'égard des droits de l'homme», in A. Riklin, H. Haug et R. Probst (éd.), Nouveau manuel de la politique extérieure suisse, Haupt, Berne, 1992, p. 231.

30. Ainsi s'exprimait Franz Blankart, alors secrétaire d'Etat et directeur de l'Office fédéral des affaires économiques extérieures, le 2 septembre 1996 devant l'Assemblée générale de l'Institut européen de Bâle.

31. Voir P. Meyer-Bisch, «L'écoéthique: interférence entre logiques économiques et logiques des droits de l'homme», in M. Borghi et P. Meyer-Bisch (éd.), Ethique économique et droits de l'homme. La responsabilité commune, Fribourg, 1998, pp. 3 et suivantes.

32. «Le développement durable se présente sous trois aspects: écologique, économique et social, qui sont équivalents et interdépendants », site du Seco, Thèmes de la politique économique extérieure suisse: le commerce et le développement durable (www.admin.ch/bawi/f/themen/umwelt/index.htm). 
Même dans les dossiers relatifs à la coopération économique, partie intégrante de la politique de développement, la logique économique fait disparaître la problématique des droits humains. Le message de 1996 concernant cette coopération lui attribue quatre objectifs: soutenir les efforts d'ajustement des pays en développement et les aider à amortir les chocs externes, faciliter l'intégration des pays en développement dans le commerce mondial, développer les capacités productives et les infrastructures, concilier les objectifs de croissance économique et de sauvegarde de l'environnement ${ }^{33}$. Ce dernier objectif reprend directement la volonté du Conseil fédéral de veiller à la préservation du milieu naturel dans l'ensemble de sa politique extérieure. Il a d'ailleurs une haute priorité: il faut «au minimum démontrer que les mesures envisagées n'ont pas d'incidence défavorable sur l'environnement ${ }^{34}$ et une collaboration est signalée avec l'Office fédéral de l'environnement, des forêts et du paysage ${ }^{35}$. Les autres objectifs de la politique extérieure de la Confédération ne sont nullement mentionnés quand la cohérence de la coopération au développement est traitée ${ }^{36}$.

L'absence de mention explicite ne signifie pas que les droits humains sont ignorés, mais rien ne garantit leur prise en compte. Dans ce contexte, le fait que l'Office fédéral des affaires économiques extérieures, maintenant intégré dans le Seco, se soit associé aux Lignes directrices pour la promotion des droits de l'homme de la DDC et affirme participer à leur mise en œuvre est un signe positif. Cela devrait faciliter la collaboration entre les divisions politiques du DFAE, la DDC et le Seco. Il est souhaitable que cette collaboration permette d'effectuer des études d'impact sur les droits de l'homme de la même manière que sur l'environnement. Il faudrait au minimum démontrer que les mesures envisagées dans le cadre de la coopération économique n'ont pas d'incidence défavorable sur la cohésion sociale, sur les droits de l'homme, sur le maintien de la paix. A côté de la préservation du milieu naturel, les autres objectifs de la politique extérieure de la Confédération doivent être pleinement pris en compte.

\section{$\square$ L'aide au retour des personnes admises provisoirement}

La Suisse n'est pas un pays d'immigration, affirme la doctrine officielle. Elle n'ouvre sa porte que pour les travailleurs très qualifiés en dehors de l'Union européenne, des pays de l'AELE et de l'Amérique du Nord. Cette forte restriction à l'immigration a pour conséquence que la seule voie d'accès au territoire suisse est la procédure d'asile. Du coup les requérants d'asile passent, aux yeux de certains, comme des personnes abusant de l'hospitalité helvétique. Cette manière de voir envenime depuis de nombreuses années le débat sur l'asile dans notre pays et conduit à une politique toujours plus restrictive. Deux grands trains de mesures illustrent cette évolution. La dissuasion à l'entrée est devenue très forte; les procédures de non-entrée en matière sur les demandes d'asile se sont multipliées avec l'arrêté fédéral urgent de 1990 puis celui de 1998; ces deux arrêtés font maintenant partie de la nouvelle loi sur l'asile approuvée par le

33. Message concernant la continuation du financement et la réorientation des mesures de politique économique et commerciale au titre de la coopération au développement du 29 mai 1996 (ci-après Message sur la coopération économique), chiffre 311.

34. Message sur la coopération économique, chiffre 311.4 .

35. Message sur la coopération économique, chiffre 312.6.

36. Les Lignes directrices Nord-Sud sont certes mentionnées, mais leur contenu n'est rappelé qu'en termes généraux. Cette relation aux objectifs généraux de la politique extérieure dans son ensemble n'est pas davantage inscrite dans l'article constitutionnel relatif à la politique économique extérieure (art. 101 de la nouvelle Constitution fédérale). 
peuple le 13 juin $1999^{37}$, qui introduit par ailleurs des limitations dans le droit de recours des requérants. Le deuxième train de mesures vise à garantir le retour effectif des requérants dont la demande est refusée. Les mesures de contrainte de 1994 permettent de détenir des personnes jusqu'à douze mois en vue d'un renvoi. L'introduction d'une clause relative à la reprise de ses propres ressortissants dans la liste des raisons pouvant inciter le Conseil fédéral à interrompre la coopération avec un pays est conforme à cette préoccupation ${ }^{38}$.

Un autre aspect des renvois concerne l'aide au retour apportée aux personnes admises provisoirement en Suisse. Un tel programme a été mis en œuvre pour la première fois en 1997 et 1998 pour les Bosniaques. Un nouveau programme a été lancé en 1999 pour les Albanais du Kosovo. Ces programmes sont menés conjointement par l'Office fédéral des réfugiés (ODR) et la DDC, le premier octroyant l'aide financière individuelle aux candidats au retour, la seconde accordant une aide à la reconstruction sur place.

Cette collaboration inédite est encore en rodage. Toujours est-il qu'elle inaugure une manière de faire qui mérite examen. Le rapport d'évaluation de l'Institut d'ethnologie de l'Université de Berne commandité par la DDC sur le programme d'aide au retour des Bosniaques a mis en évidence un conflit d'objectifs très clair ${ }^{39}$. D'un côté, le but est d'inciter les personnes admises provisoirement à quitter la Suisse. Le rapport conclut que ce but a été dans l'ensemble atteint. D'un autre côté, ce programme devait permettre aux personnes revenues dans leur pays d'y retrouver une place. Or cet objectif n'a pas été atteint. Au vu des témoignages recueillis pour cette évaluation et de ceux qui ont pu être rassemblés par la suite, on constate que les personnes qui n'ont pas pu réintégrer leur domicile d'avant la guerre ont utilisé l'argent mis à leur disposition pour survivre et sont devenues des réfugiés dans leur propre pays. Encore récemment, $\mathrm{j}$ 'ai entendu des Bosniaques remarquer que beaucoup de réfugiés rentrés d'Europe occidentale cherchent à repartir au plus vite. Autrement dit, le lent processus de recomposition d'un tissu social et économique n'a pas été pris en considération par les autorités du pays d'accueil qui voulaient voir ces personnes partir rapidement.

Les Albanais du Kosovo qui ont quitté la Suisse jusqu'à fin 1999 l'ont fait de leur plein gré; ils avaient hâte de rentrer au pays et ils ont trouvé sur place, grâce à l'aide à la reconstruction, les moyens de restaurer un tant soit peu leur demeure pour passer l'hiver. Il reste toutefois plusieurs dizaines de milliers de personnes dont l'admission provisoire prend fin au 31 mai 2000. Et à ce moment on risque de retrouver la même difficulté: faire coïncider deux rythmes temporels différents, l'urgence du départ de la Suisse et la lenteur de la reconstruction économique et sociale dans le pays d'origine.

Si l'exigence d'un départ rapide de la Suisse peut se justifier du point de vue de la politique intérieure, elle n'est par contre pas compatible avec le rythme du rétablissement de la paix et de conditions de vie permettant d'envisager sereinement un avenir dans son propre pays. L'aide au départ devrait être un investisse-

37. Voir, dans la partie «Revue» du présent Annuaire, la partie 1.3 « Votations du 13 juin sur la loi sur l'asile».

38. Communiqué du Conseil fédéral du 20 septembre 1999. Voir plus haut le dernier paragraphe de la sous-section «Les droits de l'homme dans les relations Nord-Sud » et la note 15.

39. H.-R. Wicker, C. Moser und T. Gass, Evaluation des Rückkehrhilfe- und Wiedereingliederungsprogrammes für bosnische Staatsangehörige, Bern, Januar 1998, p. 76. 
ment visant à renforcer les chances de réintégration dans la société d'origine. Elle devrait pour ce faire commencer en Suisse par l'élaboration de projets avec les personnes résidant encore dans notre pays. Elle devrait les aider à acquérir ici les compétences nécessaires pour contribuer de manière valable à la reconstruction de leur pays quitte à surseoir au départ ou à prévoir des allers-retours pendant une certaine période afin de garantir la viabilité des projets mis en place. Je pense par exemple au personnel médical ou aux enseignants admis provisoirement en Suisse. Pourquoi ne pourraient-ils pas se préparer ici en Suisse à soigner les traumatismes consécutifs à la guerre avant de rentrer au pays, se préparer à enseigner, non seulement les mathématiques ou la lecture, mais aussi les règles de la démocratie et la cohabitation entre les ethnies ? Cela implique une organisation du séjour en Suisse qui tienne mieux compte des exigences de la reconstruction psychique et sociale tout autant que matérielle dans le pays d'origine, et qui permettrait de mieux articuler les efforts diplomatiques en faveur de la paix et de la reconstruction avec la politique d'asile.

\section{PROPOSITIONS}

\section{$\square$ Un observatoire de la cohérence}

L'hypothèse de cet article est que le problème de la cohérence ou de l'incohérence des relations Nord-Sud d'un pays comme le nôtre ne résulte pas seulement de conflits d'intérêts et d'objectifs, mais est un problème culturel. Comprendre différemment la place de l'économie dans les relations internationales et participer ainsi à l'élaboration des valeurs communes à l'humanité entière, faire valoir le principe de "rétinité », dépasser les souverainetés nationales vers un Etat de droit sur le plan mondial sont quelques pistes développées pour affronter ce problème culturel. C'est à ce niveau que j'ai essayé de me situer. Mais il reste à mener une véritable analyse factuelle de l'action menée par les divers acteurs des relations de notre pays avec le «Sud». Pour ce faire, il manque encore à notre pays un véritable «observatoire de la cohérence» capable de développer les outils d'analyse permettant de cerner la problématique et de mener des enquêtes empiriques en vue de décrypter les cohérences et incohérences de l'action de la Confédération. En regroupant tant les milieux universitaires que les milieux non gouvernementaux, cet observatoire pourrait être l'outil permettant à la société civile, aux milieux économiques ainsi qu'à l'Etat de mieux comprendre les enjeux et la nécessité de la cohérence, de mieux percevoir, au-delà des intérêts à court terme divergents, la convergence à long terme des intérêts de tous les acteurs ${ }^{40}$.

Certains ont proposé de créer en Suisse, à l'image de ce qui se fait en France, une commission consultative en matière de droits humains ${ }^{41}$. L'idée est intéressante dans la mesure où cette commission permettrait de donner à la Suisse une instance capable de montrer publiquement où se situent les points forts et les points faibles dans la manière dont le gouvernement respecte les droits humains, mais elle risque de ne pas avoir les moyens de sortir d'une simple recension des

40. Pour un exemple ponctuel d'une analyse systématique, voir le dossier sur l'Indonésie publié dans l'ASTM 1995, pp. 151-232.

41. Tel Benedikt von Tscharner, dans la NZZ du 2.12.98, p. 15. 
manquements aux principes établis. Par ailleurs, il existe déjà plusieurs commissions extraparlementaires. Se poserait inévitablement un problème de partage de compétences, par exemple avec la Commission contre le racisme. Un observatoire qui aurait une assise universitaire plus forte que ne pourrait l'avoir une commission permettrait d'aller au-delà des constats et de développer les instruments d'une analyse de cohérence qui inclue tant les droits humains que les problématiques de paix, de développement durable, et l'orientation de la politique économique.

\section{$\square$ Une commission parlementaire des droits humains}

Mais une telle instance d'observation ne peut avoir un réel impact que si elle trouve des relais dans le monde politique. Or il manque jusqu'à ce jour une véritable commission, qui, au niveau des Chambres fédérales, serait à même d'examiner l'action du Conseil fédéral du point de vue de la cohérence. La Commission des affaires extérieures peut avoir cette tâche, mais il faudrait en son sein une sous-commission à même d'examiner de manière plus spécifique la cohérence du point de vue des droits humains. Car les pactes et conventions ratifiés par la Confédération comme par la Constitution imposent maintenant à la Suisse d'articuler plus fortement les divers volets de sa politique extérieure avec les droits reconnus internationalement.

\section{$\square$ Un pôle fort au sein de l'administration}

A un partenaire au sein de la société civile tel un «observatoire de la cohérence » et à une commission parlementaire doit répondre un service fédéral correspondant. Actuellement, au sein du Département fédéral des affaires étrangères, la Division politique IV (DP IV) est en charge des questions de droits de l'homme. Cette organisation a été voulue par le conseiller fédéral Flavio Cotti afin de donner davantage de visibilité à l'action de la Suisse dans ce domaine. Mais il reste encore à créer une véritable instance en charge de la cohérence de la politique extérieure de la Confédération en matière de droits humains. Elle devrait être le lieu où serait concentrée toute la compétence du Département en matière de droits humains, y compris la dimension juridique qui reste pour l'instant extérieure à la DP IV actuelle. Elle devrait ensuite être en mesure de donner un avis au sujet de l'impact de toute décision de politique étrangère - y compris dans le Département de l'économie publique - sur le domaine des droits humains, et un avis entendu. Plus encore qu'actuellement, il faut en effet que cette dimension soit déterminante dans la prise de décision. Pour cela la collaboration entre la DDC, la division en charge des droits humains, les autres divisions et directions du DFAE et le Seco doit devenir plus intensive que jusqu'à présent. A l'instar de ce qui se fait pour le Bureau de l'intégration, on pourrait même imaginer un office interdépartemental chargé de toutes les questions relatives aux droits humains dans les relations extérieures, y compris économiques.

\section{$\square$ Une politique étrangère dynamisée et reconnue}

D'une manière significative, les Lignes directrices Nord-Sud font état, à côté de la mondialisation de l'économie et d'une mondialisation des problèmes de notre $^{42}$ environnement naturel, d'une «globalisation de valeurs fondamentales:

42. Je souligne. 
le principe d'une société ouverte et démocratique, le rôle du marché en tant que principe d'ordre économique et le respect nécessaire de l'équilibre écologique $»^{43}$.

Cette «mondialisation des valeurs fondamentales $»^{44}$, bien que partielle et balbutiante, est une chance pour la Suisse. Elle lui donne l'occasion de jouer un rôle en défendant de manière pugnace et efficace les valeurs ancrées dans les plus beaux aspects de sa tradition, à savoir la tradition humanitaire. La Suisse est fière d'être le berceau et la gardienne du droit humanitaire. Elle a maintenant la chance d'étendre cette fierté aux domaines connexes, la paix et les droits humains, en défendant les valeurs dont témoigne la Charte des droits humains que la Suisse a adoptée durant les années 90. Petit pays, disposant d'une influence non négligeable à cause de la force de son économie, elle peut montrer qu'une bonne politique économique passe par un examen précis des impacts sur l'environnement et sur les droits humains, et qu'une bonne politique étrangère repose sur des valeurs orientées vers le long terme.

Mais, tout seul, il est impossible de défendre les droits de l'homme de manière cohérente. C'est pourquoi il est impensable que la Confédération reste isolée comme elle l'est aujourd'hui. Ainsi que le relevait le Conseil fédéral dans son rapport sur l'ONU de 1998, beaucoup de choses rapprochent la Suisse de l'ONU, et en particulier cette volonté de faire des droits humains une dimension transversale de toutes les actions entreprises sur le plan international. Il serait donc cohérent que la Suisse adhère enfin à l'ONU pour pouvoir y mener, non seulement en tant qu'observatrice, mais enfin de plein droit, une politique des droits de l'homme qui s'enracine essentiellement dans le dialogue et les mesures positives avant les sanctions comme ultime recours. Je crois qu'une adhésion à l'ONU motivée par la volonté de défendre les droits de l'homme et de contribuer à une ONU plus efficace dans ce domaine peut être populaire et réunir une majorité de citoyens et de citoyennes.

43. Lignes directrices Nord-Sud, chiffre 11

44. Le mot «mondialisation» en français est plus adapté. L'anglicisme «globalisation» tend à renvoyer plus étroitement à la mondialisation de l'économie. 